\title{
Autorenverzeichnis
}

\section{Hans Bach}

Dr. rer. pol., Professor an der Universität Linz

A-4045 Linz (Auhof)

Claus-Ekkehard Bärsch

Dr. phil., Privatdozent an der Gesamthochschule - Universität Duisburg

D-4100 Duisburg

Antonio Carrozza

Professeur de l'Université de Pise, Président de l'A.J.C.D.A.

Via Antonio Pisano 19

I-56100 Pisa

Harry Ebersbach

Dr. jur., Professor an der Universität Göttingen

Nikolausbergerweg

D-3400 Göttingen

\section{Günther Frohberg}

Dr. jur., Honorarprofessor an der Technischen Hochschule Aachen,

Rechtsanwalt in Düsseldorf

Wildenbruchstraße 103

D-4000 Düsseldorf-Oberkassel

\section{Ruth-Elvira Groiss}

Dr. jur., Honorarprofessor an der Technischen Hochschule Aachen;

Peter-Jordan-Straße 82

A-1190 Wien

\section{Peter Kalbe}

Dr. jur., M.C.L., Rechtsberater der Kommission der Europäischen Gemeinschaften, Brüssel

Rue de la Loi

B-1000 Brüssel

Otto Kimminich

Dr. jur., Professor an der Universität Regensburg

Killermannstraße 6

D-8400 Regensburg 


\section{Autorenverzeichnis}

Franz Klose

Ministerialdirigent a. D. im Bundesministerium für Ernährung, Landwirtschaft und Forsten, Bonn

Haus Dürffenthal

D-5352 Zülpich

\section{Manfred Köhne}

Dr. sc. agr., Professor an der Universität Göttingen

Nikolausbergerweg

D-3400 Göttingen

\section{Aimé De Leeuw}

Dr. sc. adm., Professeur extraordinaire à l'Institut des Hautes Etudes de Droit Rural et d'Economie Agricole, Paris; Vice-Président de la Commission »Cadastre et Aménagement rural « de la Fédération Internationale des Géomètres; Conseiller de la Société nationale terrienne, Bruxelles

Grote Markt 73

B-9060 Zelzate

\section{Alfredo Massart}

Incaricato nell'Università di Pisa

Piazza d'Ancona 5

I-56100 Pisa

\section{Jean Megret}

Avocat à la Cour d'Appel de Paris; Directeur de l'Institut des Hautes Etudes de Droit Rural et d'Economie Agricole, Paris; Président de l'Association Française de Droit Rural; Délégué Général du Comité Européen de Droit Rural

17 Rue Duroc

F-75007 Paris

José Luis de los Mozos

Catedrático de Derecho civil de la Universidad de

E-Valladolid

John Murray

Advocat in Edinburgh

4 Moray Place

GB-Edinburgh EH 36 DS

Jacques Prévault

Maître de Conférences à l'Université de Besançon

3 Impasse Richelieu

F-63100 Clermont Ferrand 
Juan José Sanz Jarque

Catedrático de Derecho Agrario y Sociología de la Universidad Politécnica de Madrid; Director de la Asociación Española de Derecho Agrario; Delegado Adjunto del Comité Europeo de Derecho Rural

Alberto Aquilera, Dup. 15, 7, B

E-Madrid 15

\section{Franz Schad}

Professor (em.) an der Universität Hohenheim; Ministerialdirigent a. D. im Kultusministerium Baden-Württemberg

Kornbergweg 1

D-7321 Hattenhofen

\section{Robert Scheyhing}

Dr. jur., Professor an der Universität Tübingen

Landhausstraße 13

D-7406 Mössingen

\section{Eberhard Schmidt-Aßmann}

Dr. jur., Professor an der Universität Bochum

Königsallee 63

D-4630 Bochum

\section{Flemming Tolstrup}

Dr. jur., Professor an der Universität Hellerup

Stranvey 132

DK-2900 Hellerup

\section{Edgar Tomson}

Dr. jur., Dozent an der Universität Münster

Schallstraße 6-8

D-5000 Köln 41

\section{Robert Weimar}

Dr. jur., Dr. phil., Professor an der Gesamthochschule - Universität Siegen

Hölderlinstraße 3

D-5900 Siegen 21

\section{Manfried Welan}

Dr. jur., Professor an der Universität für Bodenkultur Wien

Gregor-Mendel-Straße

A-1190 Wien 
XII Autorenverzeichnis

Wolfgang Winkler

Dr. jur., Akademischer Oberrat an der Universität Göttingen

Nikolausbergerweg

D-3400 Göttingen

Nicolaas Maarten Zijp

Dr., Sekretär des Landbouwschap, Den Haag

Ardennen 28

NL-3831 Leusden 